\title{
Numerical Synthesis of Dielectric Embedded Electronically Steerable Multiple Beam Antenna Array
}

\author{
Lei Liu ${ }^{1,2}$, Junwei Lu ${ }^{2}$, Shiyou Yang ${ }^{1}$ \\ ${ }^{1}$ College of Electrical Engineering, Zhejiang University, China \\ ${ }^{2}$ Faculty of Engineering and Information Technology, Griffith University, Australia
}

\begin{abstract}
Dielectric embedded electronically steerable multiple beam antenna array is a potential candidate in mobile wireless communications. However, its synthesis is challenging due to the existence of the large conflicting objectives and infeasibility of analytical synthesis techniques. In this regard, an extended evolutionary algorithm is proposed and tested using different case studies.
\end{abstract}

\section{Models And Methodologies}

Based on the directional Yagi-Uda antennas, the electronically steerable multiple beam (ESMB) antenna array has recently been developed as a potential adaptive antenna in mobile wireless communications. Moreover, by using shortcircuited monopole elements, an ESMB antenna array can operate as a corner reflection antenna with further enhancements in the directivity. However, without the employment of dielectric embedded techniques, this exclusive advantage can not be fully utilized in mobile wireless communications due to its bulky size [1]. Fig. 1 shows the configuration of a dielectric embedded ESMB (DE-ESMB).

The size reduction of a DE-ESMB antenna array because of the introduction of permittivity $\varepsilon_{r}$ can be estimated by

$$
l_{r}=l_{o} / \sqrt{\varepsilon_{r}}
$$

where; $l_{o}$ and $l_{r}$ are the lengths of an ESMB antenna array in vacuum and dielectric, respectively.

In practical implementations, the whole space cannot be filled with dielectrics. Therefore, discontinuities exist at the edges of dielectrics, which will result in inapplicability of both (1) and available analytical synthesis techniques. In this regard, one has to resort to numerical techniques.

The synthesis of a DE-ESMB antenna array is formulated as a many-objective optimization problem, i.e., a trade-off is made for more than triple objectives. The objectives include the reduction of the physical size, the improvement of the gain, the minimization of the reflection coefficient $S_{11}$ and its sensitivity at the operation frequency.

Although the vector evolutionary algorithm has become a standard for solving multi-objective designs, the selection pressure becomes so weak that it can not catch hold of the Pareto front in many-objective cases. Hence one extends the Multiple Single Objective Pareto Sampling-II algorithm (MSOPS-II) [2] to synthesize a DE-ESMB antenna array. With the help of a group of target vectors, i.e., the direction vectors in the hyperspace, this aggregation based algorithm can effectively uphold the selection pressure. However, those target vectors can not be always properly distributed using the original updating mechanism. In this regard, a crowding operation is proposed to measure the diversity of the target vec-

tors, which takes the position of the original updating mechanism. In addition, the exhaustive searches in a huge parameter space will inevitably slow down the convergence speed of MSOPS-II. To avoid unnecessary searches, the preference is added using the concept of goals:

$$
f_{j}(x) \in\left[t_{j}^{l}, t_{j}^{u}\right]
$$

where; $f_{j}(x)$ is the $j^{\text {th }}$ objective function; $t_{j}^{l}, t_{j}^{u}$ are, respectively, the minimum and maximum bounds of the preferences of the objective.

By means of this preference approach, the searching space is reduced significantly.

\section{RESULTS AND DISCUSSIONS}

The extended MSOPS algorithm is firstly validated by solving test functions ZDT1 and ZDT2. The goals are set as, respectively, as $f_{1}, f_{2}>0.3$ for ZDT1, and $f_{1}, f_{2}<0.7$ for ZDT2. The searched fraction of the Pareto front is shown in Fig. 2. Obviously, the proposed algorithm can uniformly sample the specified fractions of the Pareto front of a many-objective design problem. The proposed method is then used to synthesize a typical DE-ESMB. The optimized results are summarized as: both the radius and height are reduced within $8 \mathrm{~cm}$, the gain is $2.5 \mathrm{~dB}$, and the $S_{11}$ is $-9 \mathrm{~dB}$. Therefore, the feasibility and advantages of the proposed synthesis techniques for solving DE-ESMB are positively confirmed.

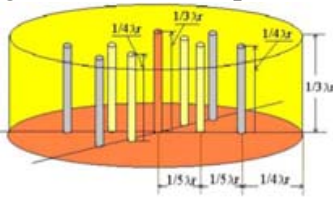

(a)

Fig.1. DE-ESMB:(a) configuration (b)circuit connection.
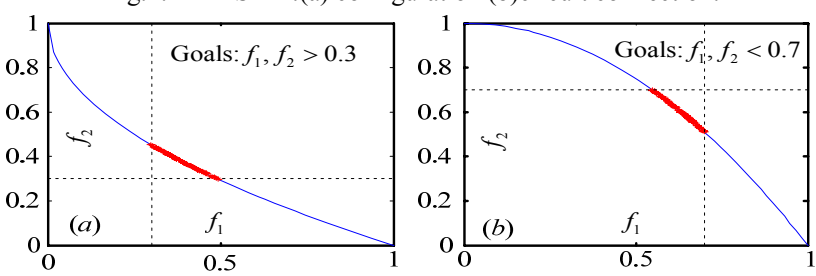

Fig. 2. The searched Pareto front of (a) ZDT1 and (b) ZDT2.

\section{REFERENCES}

[1] Junwei Lu, Iwashita T., "Dielectric embedded electronically steerable multiple beam (DE-ESMB) antenna for mobile wireless computation system," Asia-Pacific Conference on Environmental Electromagnetics, pp. 278-281, 2003.

[2] Hughes E. J., "MSOPS-II: A general purpose Many-Objective Optimizer," Congress on Evolutionary Computation, pp.3944-3951, 2007. 